IPOS scores on initial assessment at one centre $(\mathrm{N}=15)$, recorded the main symptoms as breathlessness, weakness and anxiety. Family anxiety was the highest scoring aspect of the IPOS. The medical management at the end-of-life was, however, generally uncomplicated; the total opioid (oral morphine equivalent) and benzodiazepine doses administered in last 24 hours before death were relatively low, median dose (range) $31.25 \mathrm{mg} \quad(5-160 \mathrm{mg})$ and $15 \mathrm{mg} \quad(0-30 \mathrm{mg})$ respectively.

Conclusions Should a further surge of COVID-19 cases occur, many cancer patients dying with COVID-19 could be appropriately managed by non-specialist physicians supported by comprehensive guidelines, ward-based teaching and specialist palliative care input for more complex cases. High levels of family anxiety could be improved by enhanced family support and communication about end-of-life wishes and priorities; healthcare services should have a particular focus on supporting the shadow pandemic of loss.

\section{EVALUATING THE ROLE OF THE ROYAL FREE LONDON NHS FOUNDATION TRUST PALLIATIVE CARE TEAMS DURING THE COVID-19 PANDEMIC}

Rory Carrigan, Sophie Cakebread, Louise Schofield, Nicola Henawi, Jo Wilson. Royal Free London NHS Foundation Trust

\subsection{6/spcare-2021-PCC.45}

Background The SARS-Cov-2 pandemic resulted in a rapid and unprecedented shift in the number of patients admitted to hospitals. In this trust palliative care provide a 9-5, 7 day/ week liaison service. We evaluated the role of the palliative care services during the peak of the pandemic.

Methods We conducted a retrospective analysis of the demand on palliative care team (PCT) at the two acute hospital sites; Royal Free Hospital (RFH) and Barnet Hospital (BH). Trust referral data was recorded for a 6 -week period between 18/03 and 29/04, 2020. Patient outcomes were documented on a standardised Excel-database. Clinical notes were audited at random to ensure quality of data capture.

Results During the period studied there were 597 deaths between both sites, $393(66 \%)$ of which were documented as Covid-19 related. BH referred 178 patients to the PCT, of which 90\% were Covid-19 related. RFH referred 99 patients, of which 58\% were Covid-19 related. Clinical support provided to the wards caring for the patients was predominately in person at $\mathrm{BH}(97 \%)$ and via telephone at RFH (76\%), with an average time to death after referral of 1.9 days at $\mathrm{BH}$ and 2.8 days at RFH. Approximately $16 \%$ of patients at both sites were discharged to other services for on-going care or end-of-life-care at home. The majority of pharmacological interventions were as-required medication $(>85 \%)$ with fewer patients than anticipated needing a syringe driver $(<60 \%)$. Clinical notes confirmed that most patients became symptomatic quickly, died rapidly after referral and communication with families via telephone was well documented.

Conclusions The two PCTs had different clinical experiences, this can be explained by the populations that each site serves, the structures within the teams and their physical location in relation to the wards. Excellent patient outcomes remained the same suggesting that both PCTs adapted well, with further shared learning planned.

\section{A REVIEW OF CARDIOPULMONARY RESUSCITATION (CPR) AND TREATMENT ESCALATION PLAN (TEP) DECISION-MAKING IN AN ACUTE LONDON TRUST DURING THE COVID-19 PANDEMIC - AN AUDIT-BASED STUDY}

Amelia Parker, Eleanor Manners, Lucy Gudge, Ben Hardy, Dijay Dave, Kathleen Bonnici, Ruth Caulkin. Chelsea and Westminster NHS Foundation Trust

\subsection{6/spcare-2021-PCC.46}

Introduction National guidance early in the COVID-19 pandemic encouraged frontline hospital staff to have discussions and make early decisions with patients regarding appropriateness of CPR and other medical treatments if they were to deteriorate.

An audit of CPR and TEP decisions was carried out in our trust at the pandemic peak and four weeks later compared to data from the previous year.

Methods Admission data was used to determine the peak of COVID-19 admissions. Electronic records of all adult inpatients (excluding maternity and Emergency Department) were reviewed and any completed CPR decisions and TEPs on the $2 / 4 / 20$ (peak) and 30/4/20 analysed using the same criteria as previous CPR and TEP audit.

Results and Discussion There was a 3 -fold increase in patients with a CPR decision at the peak (77\%) of the pandemic, compared with pre-pandemic (26\%), with a marked increase in number of decisions 'For CPR' from 1\% pre-pandemic to $33 \%$ at peak. However, this increase in number of CPR decisions reduced 4-weeks post the peak to $58 \%$ as the pressure on admissions and ICU beds decreased.

The number of patients with a 'No CPR' decision with a TEP increased from $59 \%$ pre-pandemic to $88 \%$ at the peak suggesting an increased focus on reviewing benefits of treatments such as ventilation together with a CPR decision. This declined to $53 \%$, below pre-pandemic levels, at 4-weeks.

$70 \%$ of CPR decisions were made within 2 days of admission at the peak which declined to $65 \%$ 4-weeks later.

Conclusions At the peak of COVID-19 related admissions, the data set shows a marked increase in CPR decisions and completed TEPs compared to pre-pandemic baseline, indicating that the importance of such conversations and prioritisation of resources became a focus for healthcare professionals. The increase was not, however, sustained. Maintaining focus on early decision-making remains the challenge.

\section{DISCUSSIONS OF PREFERRED PLACE OF DEATH IN SECONDARY CARE DURING THE COVID-19 PANDEMIC}

Ruth Porther, Karishma Tailor, Prateek Choudhary, Julia McLaughlin, Shruti Lakra. Aneurin Bevan Health Board

\subsection{6/spcare-2021-PCC.47}

Objectives Achieving Preferred Place of Death (PPD) may form part of a 'good death' for some patients and loved ones. In many circumstances, it may not be possible to facilitate a death outside of hospital during the COVID-19 pandemic but this does not exclude the need for constructive discussions of PPD in order to address this. The aim of this audit is to determine whether PPD is being discussed in a secondary care setting alongside Treatment Escalation Plans (TEPs) and explore influencing factors. 
Methods Notes of 20 patients who died in Aneurin Bevan Health Board during the COVID-19 pandemic were audited for discussions of PPD and TEPs.

Results Even in cases where patients remained stable after the decision for supportive care was made, only in one case was PPD discussion documented. In contrast, in almost all cases there was a DNAR in place that was also discussed with the patient and/or family.

Conclusions Discussing TEPs is not necessarily the equivalent of robust joint decision-making. As we continue to manage dying patients during the pandemic, factors influencing PPD discussions in view of the wider context of end-of-life discussions should be considered. There is scope for research into how the COVID-19 pandemic has influenced or changed the quality of end-of-life discussions in secondary care to ensure patient-centred care moving forward.

\section{REMEMBER ME: SUPPORT THROUGH REMEMBRANCE DURING THE COVID19 PANDEMIC}

Ruth Pryce, Rachel Morris, Sarah Stanley. Marie Curie Hospice Liverpool

10.1136/spcare-2021-PCC.48

Background The COVID19 pandemic has highlighted a greater need for spiritual support and remembrance in a difficult time when usual comforts associated with end of life care have been stripped away. Marie Curie Hospice Liverpool have worked to develop unique ways to continue support through spiritual care and remembrance using technology and links to our local communities.

Methods There have been many challenges throughout the pandemic including the closure of the hospice to visitors, meaning for patients that face to face contact with relatives or their own faith leaders was no longer possible. Our chaplain continues to provide spiritual care for patients and carers, whilst creating remembrance opportunities. Embracing technology and the support of the local community has been vital in enabling patients to receive the best possible spiritual care. An example of this is the engagement of local church groups supporting the hospice by providing knitted hearts to keep patients and relatives connected. Remembrance opportunities have been created, including: online memorial services, use of technology to allow patients and families to come together for prayer, weekly email reflections to support staff wellbeing, and developing the chapel into an 'escape room' providing space for reflection during difficult times.

Results Results are positive. Patients and carers report feeling supported spiritually in a difficult time, which has been essential to their care. Remembrance work via online platforms has allowed our hospice and local community to be supported through bereavement and feedback from carers, local community groups and staff has been positive and complimentary.

Conclusions Our work has demonstrated that despite the challenges presented during the pandemic, spiritual care and remembrance continues to provide comfort to those affected. Developing unique ways to continue supporting people has proven to be successful. Work is ongoing. We are eager to innovate and continue providing support in these difficult times.

\section{EFFECTIVENESS OF A PALLIATIVE CARE RESOURCE TOOLKIT FOR COVID 19 FOR LOW AND MIDDLE INCOME COUNTRIES (LMICS) ON HEALTH CARE WORKERS KNOWLEDGE AND CONFIDENCE LEVELS}

Sunitha Daniel, Chitra Venkateswaran, Rajashree K Chittazhathu, Smriti Rana, Mhoira Leng, PalliCovidKerala Study Group. National Health Mission, General Hospital Ernakulam, Kochi, India Believers Church Medical College, Thiruvalla, Kerala, India Amrita Institute of Medical Sciences, Amrita University, Kochi, India Wolfson Palliative Care Research Centre, University of Hull

\subsection{6/spcare-2021-PCC.49}

Background Integrated palliative care (PC) has an important role in supporting those affected by the global COVID 19 pandemic. Communication and goals of care, symptom control and holistic support is needed particularly for patients and families living with multi-morbidity and populations in isolation and lockdown. Equipping health care workers (HCW) with core PC competencies is essential and often lacking. Building on models of integrated PC and effective response to humanitarian emergencies in Kerala, we developed and disseminated a Palliative Care in COVID-19 Resource Toolkit for LMICs comprising an e-book, webinars and ECHO platform interactive sessions for HCW.

Objective To evaluate the impact of the Resource Toolkit on the knowledge and confidence levels of HCWs.

Methods Participants registered for training package completed a pre and post course questionnaire with eighty percent attendance along with giving narrative feedback. Data collected from June to September 2020.

Results A total of 388 participants from 8 countries including 24 Indian states; 27\% male; median age 33 (20-65); 46\% nurses \& 27\% doctors. There is statistically significant improvement in all factors assessed from pretest to post test. $(\mathrm{p}=0.000))$ Mean difference in knowledge \& confidence in communication, goal setting, physical symptoms management, distress management and EOLC are as follows: 2.57 CI 95\% ; (2.21 to 2.93), 2.34 CI 95\%; (1.99 to 2.68), 2.72 CI 95\%; (2.36 to 3.07 ), 2.55 CI 95\%; (2.20 to 2.90), 2.42 CI 95\%; (2.05 to 2.79), 2.38 CI 95\%; (2.01 to 2.75), 2.88 CI 95\%; (2.51 to 3.24$), 2.63$ CI $95 \%$; (2.27 to 2.99$)$, 3.01 CI $95 \%$; (2.65 to 3.38$), 2.76$ CI $95 \%$ (2.39 to 3.13 ).

Conclusion The Toolkit when combined with online interaction can support the integration of PC competencies in health care workers many of whom have no previous exposure to PC. Narrative feedback also supported the place of a safe forum to share. Further study is planned to assess the educational impact on practice.

Funding Self-funded.

\section{VIEWING THE DECEASED PERSON: BEREAVED FAMILY UPTAKE OF A NEW SERVICE IN THE WAKE OF COVID- 19 RESTRICTIONS}

Wendy M Walker, Ruth Horton, Jenny Jones, Julie Morrell, Elaine Roberts. The Royal Wolverhampton NHS Trust

\subsection{6/spcare-2021-PCC.50}

Background Care after death includes supporting the bereaved. Evidence suggests that opportunity to view the deceased person is helpful to grieving families. During the COVID-19 pandemic, imposed restrictions to family presence challenged 